\title{
A novel Transcript is Up-Regulated by Fasting in the Hypothalamus and Enhances Insulin Signalling
}

B. Chai, J.-Y. Li, D. Fritze, W. Zhang, Z. Xia and M. W. Mulholland

Department of Surgery, University of Michigan Medical School, Ann Arbor, MI, USA.

\section{Journal of Neuroendocrinology}

Correspondence to:

Michael W. Mulholland, Department of Surgery, University of Michigan Medical School, 2101 Taubman Center, 1500 E. Medical Center Drive, Ann Arbor, MI 48109-0346, USA (e-mail:micham@med.umich.edu).
A transcript of unknown function, regulated by fasting and feeding, was identified by microarray analysis. The transcript is up-regulated in the fasting state. An 1168-bp cDNA was cloned from rat hypothalamus and sequenced. This sequence is consistent with adipogenesis down-regulating transcript 3 (AGD3) (also known as human OCC-1) mRNA. A protein sequence identical to AGD3 was determined by mass spectrometry. In the rat brain, AGD3 mRNA is distributed in the arcuate nucleus, ventromedial hypothalamus, amygdaloid nuclei, hippocampus, and somatic cortex. Double in situ hybridisation showed that AGD3 mRNA is co-localised with pro-opiomelanocortin and neuropeptide $Y$ in arcuate nucleus neurones. AGD3 binds with insulin receptor substrate 4 and increases insulin-stimulated phospho-Akt and regulates AMPactivated protein kinase and mammalian target of rapamycin downstream target S6 kinase phosphorylation.

Key words: adipogenesis down-regulating transcript 3 , insulin, proopiomelanocortin, neuropeptide $Y$ doi: 10.1111/j.1365-2826.2012.02378.x
Obesity and being overweight represent major public health problems. These conditions affect morbidity and mortality as factors contributing to diabetes mellitus, cardiovascular disease, pulmonary disease, osteoarthritis and several types of cancers. Research has established the hypothalamus as a crucial site in controlling body weight. Peripherally produced hormones such as insulin, as well as nutrients such as glucose and fatty acids, communicate energy status to hypothalamic centres (1-3). The structure of the hypothalamic arcuate nucleus (ARC) is consistent with its role as an integrative centre in energy homeostasis. Neuroanatomical studies have identified two distinct populations of ARC neurones (4). The first population expresses the orexigenic neuropeptide $Y$ (NPY) and agouti-related peptide. The second group of neurones expresses anorexigenic neuropeptides cocaine- and amphetamine-related transcript (CART) and pro-opiomelanocortin (POMC) $(4,5)$.

Gene analysis using microarrays and high-throughput sequencing has been utilised extensively as a platform for biological exploration, including conditions associated with obesity. Using this approach, a previous report from our laboratory confirmed the differential expression of several neuropeptides associated with fasting and obesity $(6,7)$. The study also suggested that intracellular regulatory genes are involved in energy homeostasis and are differentially regulated during fasting and feeding states. Over the past decade, microarray studies of cDNAs have revealed thousands of novel transcripts (8). When the functional roles have been unclear, these have been designated as transcripts of unknown function (TUF). It is widely assumed that the majority of TUFs are noncoding RNAs $(9,10)$. These noncoding RNAs may regulate gene function as microRNAs, as small nucleolar RNAs or through noncoding repression of transcription factors (10). We identified a TUF as being strongly up-regulated in the rat ARC by fasting. In the present study, this TUF was identified as derived from an mRNA of $1.2 \mathrm{kbp}$ identical to the previously described overexpressed in colon cancer1 (11) and, recently, adipogenesis down-regulating transcript 3 (AGD3) (12). The AGD3 mRNA has an open-reading frame that produces a 63-amino acid peptide. AGD3 is expressed in brain areas relevant to feeding behaviour and is involved in cellular signalling pathways that relate to energy metabolism and may be involved in controlling feeding behaviour.

\section{Materials and methods}

Antibodies and reagents

Anti-Myc antibody-conjugated agarose was purchased from Bethyl Laboratories (Montgomery, TX, USA). Anti-Myc monoclonal antibody was purchased from BD Bioscience (Palo Alto, CA, USA). Protein A SepharoseTM CL-4B was obtained from GE Healthcare (Uppsala, Sweden). Anti-human 
insulin receptor substrate 4 (IRS4) antibody (EP907Y) was obtained from Abcam (Cambridge, MA, USA). Phospho-Akt (Thr308) antibody, Akt antibody phospho-AMP-activated protein kinase (AMPK)a (Thr172) antibody, AMPKa antibody, phospho-S6 (Ser235/236) antibody and S6 ribosomal protein antibody were obtained from Cell Signalling Technologies (Beverly, MA, USA).

\section{Cell culture}

Human embryonic kidney (HEK293) cells were cultured in Dulbecco's modified Eagle's medium (DMEM) with 10\% foetal bovine serum (FBS) with $100 \mathrm{U} / \mathrm{ml}$ penicillin and $100 \mu \mathrm{g} / \mathrm{ml}$ streptomycin. Human neuroblastoma (SK-N-AS) cells were obtained from ATCC (Manassas, VA, USA) and were cultured in ATCC-formulated DMEM with 1\% non-essential amino acids, 10\% FBS, $100 \mathrm{U} / \mathrm{ml}$ penicillin and $100 \mu \mathrm{g} / \mathrm{ml}$ streptomycin. Human colorectal adenocarcinoma (CACO-2) cells were obtained from Sigma-Aldrich (St Louis, MO, USA) and were cultured in Eagle's minimal essential medium. with 2 mm glutamine, 1\% non-essential amino acids and 10\% FBS. Mouse hypothalamus (GT1-1) cells were cultured in DMEM with 10\% FBS containing $100 \mathrm{U} / \mathrm{ml}$ penicillin and $100 \mu \mathrm{g} / \mathrm{ml}$ streptomycin.

\section{Animals and experimental protocol}

Male Sprague-Dawley rats (Harlan Laboratories, Madison, WI, USA) weighing 280-300 g were used to study AGD3 expression. Rats were housed in accordance with the University of Michigan Unit of Laboratory Animal Medicine guidelines. Five rats were assigned to the fed group and five rats to the fasted group. Animals in the fed group were allowed free access to regular rat chow and water. Animals in the fasted group were food-deprived $48 \mathrm{~h}$ but were allowed free access to water. At the end of 48-h period, animals were sequentially euthanised. Whole brain was removed and frozen. A 2-mm thick section from Bregma -2 to $-4 \mathrm{~mm}$ was sliced. The ARC was punched out using a Stoelting brain punch set (Stoelting Co., Wood Dale, IL, USA). Then, total RNA was isolated from the samples.

Reverse transcription quantitative PCR (RT-QPCR) was used to examine changes in rat AGD3 expression. Reverse transcription was performed using the SuperScript One-Step RT-PCR System with Platinum Taq DNA Polymerase (Invitrogen, Carlsbad, CA, USA). Q-PCR primers were: AGD3: 5'-CCG ACA GGA GCA GT AAA GAT G-3/5'-CT CCA AAT TCA CGA GCA AGC/Probe: 5'-56-FAM/AGA TAA CGC AAT GGC TGG ATA CCC G/36-TAMSp/-3' and GAP DH: 5'-ACC ACA GTC CAT GCC ATC AC/5'-CAG CTC TGG GAT GAC CT GCl 5'-/Probe: 56-FAM/CAC AGC CTT GGC AGC ACC AGT GGA/36-TAMTph/-3'. GAPDH primers were used as an internal control.

To analyse AGD3 protein expression in ARC, another set of ten rats, five fed and five fasted, were used. Animals were euthanised. The brain was removed and a $2 \times 2 \times 2 \mathrm{~mm}$ cube was directly dissected from the ARC area. The tissue was homogenised in lysis buffer and supernatant was subject to western blotting.

\section{Cloning of AGD3 cDNA}

Our gene microarray study demonstrated that an expressed sequence tag (EST) was up-regulated by fasting. The EST (Gene Bank accession number AA925734) was a 189-bp segment. To identify this gene, the EST sequence was examined using the NCBI database. By aligning the EST sequences with overlapping cDNA segments, it was extended to a 1215-bp cDNA sequence. Two pairs of PCR primers linked to the EcoRI and Kpnl enzyme sites were used to clone two portions of the sequence from rat hypothalamus by RTPCR. One pair was 5'GGA ATT CCG GGT GGC GCG ATG GGT TGC/5'GGG GTA CCA GTC TCGT CAG CTT CCT TCT ATG, covering from 117 to 529; another pair was 5'GGA AT CAC GCA ATG GCT GGA TAC CCG AGA G/5' GGG GTA CCG TTG CT ACA CTG CCG TCAT, covering from 329 to 1168. The PCR products were sequenced.

\section{AGD3 antibody production}

Polyclonal antibodies against the rat AGD3 sequence were generated and purified by affinity chromatography using a custom service from Invitrogen. The specificity of the antibodies was characterised using the myc-AGD3 fusion protein.

\section{Construction of expressing AGD3 vector and its small interfering (si)RNAs}

\section{AGD3-pcDNA3.1}

The sequence 117-529, which was cloned by RT-PCR from the rat hypothalamus and has an open-reading frame coding a 63 amino acids protein, was subcloned into the pcDNA3.1 using the EcoRI and Kpnl sites. The Myc tag was fused to the N-terminus of AGD3. The correct frame of the construct was confirmed by DNA sequencing.

\section{AGD3 siRNAs}

Three pairs of double-stranded siRNAs targeting the human overexpression in colon cancer-1 (OCC-1) gene were used. Their sequences were: (i) $5^{\prime}$-CGA AAG ACA AGU UAA CAA ACU GUC A/5'-UGA CAG UUU GUU AAC UUG UCU UUC GUA; (ii) 5'-AGC AGC CAA AGA UGU AAC AGA AGA A/5'-UUC UUC UGU UAC AUC UUU GGC UGC UCC (IDT); and (iii) 5'-CCC UGG GUA CAU AUU GUU GTT/5'-CAA CAA UAU GUA CCC AGG GT. Lipofectamine RNAiMax was used to transfect siRNAs into cells in accordance with the manufacturer's instructions (Invitrogen). After $72 \mathrm{~h}$ of transfection, cells were lysed and supernatants were analysed further.

\section{Identification of the AGD3 transcript}

A Pierce Direct IP Kit (Pierce Biotechnology, Rockford, IL, USA) was used to pull down AGD3 protein from CACO-2 cells. Briefly, AGD3 antibody was linked to agarose beads and incubated with cell lysates. After overnight incubation at $4{ }^{\circ} \mathrm{C}$, beads were rinsed thoroughly with wash buffer, and AGD3 was eluted. Eluates were resolved on 16.5\% Tris-Tricine gels (Bio-Rad, Hercules, CA, USA). Gels were stained with Bio-Safe Coomassie Blue stain solution. Protein bands recognised by western blotting directed against antiAGD3 antibody at the 7-kDa position were subjected to mass spectrometry analysis.

The protein identification was conducted at the Protein Structure Facility at the University of Michigan (Ann Arbor, MI, USA). Bands of interest were excised and the proteins digested in situ with trypsin (Promega, Madison, WI, USA). Digests were analysed by capillary high-performance liquid chromatography-electrospray ionisation tandem mass spectrometry (HPLCESI-MS/MS) on a Thermo Fisher LTO Orbitrap Velos mass spectrometer (Thermo Fisher Scientific Inc., Waltham, MA, USA) fitted with a New Objective Digital PicoView 550 NanoESI source (New Objective, Inc., Woburn, MA, USA). On-line HPLC separation of the digests was accomplished with an Eksigent/AB Sciex NanoLC-Ultra 2-D HPLC system (Eksigent, Dublin, CA, USA): column, PicoFrit (New Objective, Inc.; inner diameter $75 \mu \mathrm{m}$ ) packed to $15 \mathrm{~cm}$ with $\mathrm{C} 18$ adsorbent (Vydac, Hesperia, CA, USA; 218MS $5 \mu \mathrm{m}$, $300 \AA$ ). Precursor ions were acquired in the Orbitrap in centroid mode at 60000 resolution ( $\mathrm{m} / \mathrm{z} 400)$; data-dependent collision-induced dissociation (CID) spectra of the six most intense ions in the precursor scan above a set 
threshold were acquired at the same time in the linear trap. Mascot (Matrix Science Inc., Boston, MA, USA) was used to search the uninterpreted CID spectra against the SwissProt database (SwissProt_2011_03.fasta). Crosscorrelation of the Mascot results with X! TANDEM (http://www.thegpm.org/tandem/) and determination of protein and peptide identity probabilities were accomplished using SCAFFoLd (Proteome Software, Inc., Portland, OR, USA). The thresholds for acceptance of peptide and protein assignments in SCAFFOLD were $95 \%$ and $99.9 \%$, respectively.

\section{In situ hybridisation}

In situ hybridisation was conducted using a modification of a previously described protocol (6). Briefly, a 416-bp PCR generated fragment of the rat AGD3 was subcloned into pBluescript SK (Stratagene, La Jolla, CA, USA). The sense primer was: 5'-ACA AGA GGA GAA ACT ACG GAG GAG-3'. The antisense primer was $5^{\prime}$-TGG CCA GGA GGG AAA ACA C-3'. The NPY CDNA (Al045437) is in PT7T3D-PAC (Invitrogen). The rat POMC plasmid construct consists of an 833-bp insert in pGEM4Z (Promega).

The ${ }^{35}$ S-labelled antisense and sense AGD3 RNA probes, and digoxigeninlabelled antisense and sense NPY and POMC RNA probes, were generated using standard in vitro transcription methodology.

For single-label in situ hybridisation, the sections were hybridised with antisense ${ }^{35}$ S-labelled AGD3 riboprobe. For dual label in situ hybridisation, the sections were hybridised with antisense digoxigenin-labelled NPY or POMC and ${ }^{35}$ S-labelled AGD3 riboprobes.

Male Sprague-Dawley rats were anaesthetised with ketamine/xylazine and perfused via the ascending aorta with $200 \mathrm{ml}$ of phosphate-buffered saline (PBS), followed by $200 \mathrm{ml}$ of $4 \%$ paraformaldehyde in PBS. The brain was postfixed for $16 \mathrm{~h}$ then transferred to $20 \%$ sucrose (20\% sucrose in PBS with $0.02 \%$ sodium azide) for 5 days at $4{ }^{\circ} \mathrm{C}$. The brain was embedded with 20\% sucrose and Tissue-Tek OCT (2:1) and coronal sections of $14 \mu \mathrm{m}$ were cut on a cryostat. The sections were dried overnight at room temperature and were stored at $-80^{\circ} \mathrm{C}$ until further processing.

Co-localisation was considered if a cluster of more than 20 silver grains were above POMC or NYP positive cells.

\section{Dual immunohistochemistry}

The sections were rinsed in PBS, $0.5 \%$ triton $X-100$ and blocked in 10\% serum, and then incubated with primary antibodies [rabbit anti-AGD3 (dilution $1: 150$ ) and goat anti-POMC (dilution $1: 100)]$ overnight at $4{ }^{\circ} \mathrm{C}$. For AGD3 staining, biotinylated horse anti-rabbit secondary antibody was used and then visualised with FITC488 (green)-conjugated avidin. To visualise POMC, Texas Red-conjugated donkey anti-goat secondary antibody was used. Nuclei were stained with 4',6-diamidino-2-phenylindole dihydrochloride (Invitrogen).

\section{Co-immunoprecipitation}

HEK293 cells were transfected with myc-AGD3 pcDNA3.1 using Lipofectamine 2000 in accordance with the manufacturer's instructions (Invitrogen) and incubated for $48 \mathrm{~h}$. Transfected cells were lysed with PK buffer $(50 \mathrm{~mm}$ HEPES, pH 7.5, $150 \mathrm{~mm} \mathrm{NaCl}, 1.5 \mathrm{~mm} \mathrm{MgCl}, 1 \mathrm{~mm}$ EGTA, $1 \mathrm{~mm} \mathrm{Na} \mathrm{VO}_{4}$, $50 \mathrm{~mm} \mathrm{NaF}, 1 \%$ Triton X-100, 10\% glycerol) and briefly sonicated. The supernatants of the cell lysates were incubated with protein A or anti-mycconjugated beads for $24 \mathrm{~h}$ at $4{ }^{\circ} \mathrm{C}$. The beads were washed six times, and the immunoprecipitants were eluted with sodium dodecylsulphate (SDS) sample buffer at $95{ }^{\circ} \mathrm{C}$, resolved on by SDS-polyacrylamide gel electrophoresis (PAGE), and stained with the Coomassie Blue stain solution (Bio-Rad). Protein bands missing from the protein A bands were excised from the gel. The gels were subject to mass spectrometry analysis.
To confirm the interactions between AGD3 and IRS4, which were revealed by the protein identification analysis, eluates were resolved on SDS-PAGE gel; the gels were used for western blotting with anti-IRS4 antibody. For the co-immunoprecipitation of endogenous IRS4 from hypothalamus, rat hypothalamus tissue was homogenised in lysis buffer and the supernatant was used for immnoprecipitation with protein A and anti-AGD3 antibody. IRS4 was detected with anti-IRS4 antibody.

In the pull down of AGD3 using IRS4, HEK293 cells were transfected p3xFlag-IRS4-CMV-10 plasmid DNA (13) along with myc-AGD3 pcDNA3.1 DNA. Forty hours after transfection, cells were disrupted by sonication in Iysis buffer and Flag-IRS4 was immunoprecipitated using anti-Flag-M2 antibody conjugated agarose (Sigma). After extensive washing, the beads were resuspended in SDS-PAGE loading buffer and heated at $85^{\circ} \mathrm{C}$ for $5 \mathrm{~min}$. The protein was resolved with SDS-PAGE and transferred to polyvinylidene membranes. The membranes were blotted with either anti-myc, or anti-Flag antibody.

\section{Statistical analysis}

For Western blots, analysis of densitometry was performed using Kodak 1D 3.6 software (Eastman Kodak, New Haven, CT, USA). Data were analysed using GRAPHPAD PRISM, version 4.0 (Graphpad Software, San Diego, CA, USA), and expressed as the mean \pm SEM. Differences were analysed by unpaired two-tailed Student's t-test. $P<0.05$ was considered statistically significant.

\section{Results}

\section{Up-regulation of AGD3 in the rat ARC by fasting and obesity}

Gene profiles of the rat ARC were investigated in conditions of fasting and feeding by utilising microarray analysis (6). This analysis demonstrated 118 mRNA species that were increased by fasting relative to the fed state. An EST (gene bank number AA925743) was significantly increased in the fasted group. Quantitative PCR confirmed that this EST mRNA was increased two-fold in fasting animals relative to those in the fed group (Fig. 1A).

We also examined AGD3 transcript expression in the genetically obese Zucker rat ( $\mathrm{fa} / \mathrm{fa})$ and the lean Zucker rat. The ARC RNA was obtained from our previous experiment (6). Compared to the lean Zucker rat, AGD3 mRNA was up-regulated in the ARC of the fa/fa rat (Fig. 1B).

\section{Cloning the open-reading frame of rat AGD3}

To identify this gene, the EST sequence was examined using the NCBI database. By aligning the EST sequence with overlapping DNA segments, it was extended to a 1215-bp DNA sequence. Two pairs of PCR primers were used to clone two portions of the sequence and a segment of 1052 bp was confirmed by DNA sequencing. This rat sequence has high homology with mouse and with the human OCC-1 gene (11). This sequence also matches a transcript of unknown function, the AGD3, as reported by Kikuchi et al. (12). The 1052-bp contains several open-reading frames. An open-reading frame that can translate a 63 amino acid protein was proposed as a hypothetical protein. The rat hypothetical protein has 86\% and 97\% sequence identity with the human (NP_001138671) and mouse (NP_001138670), respectively. 

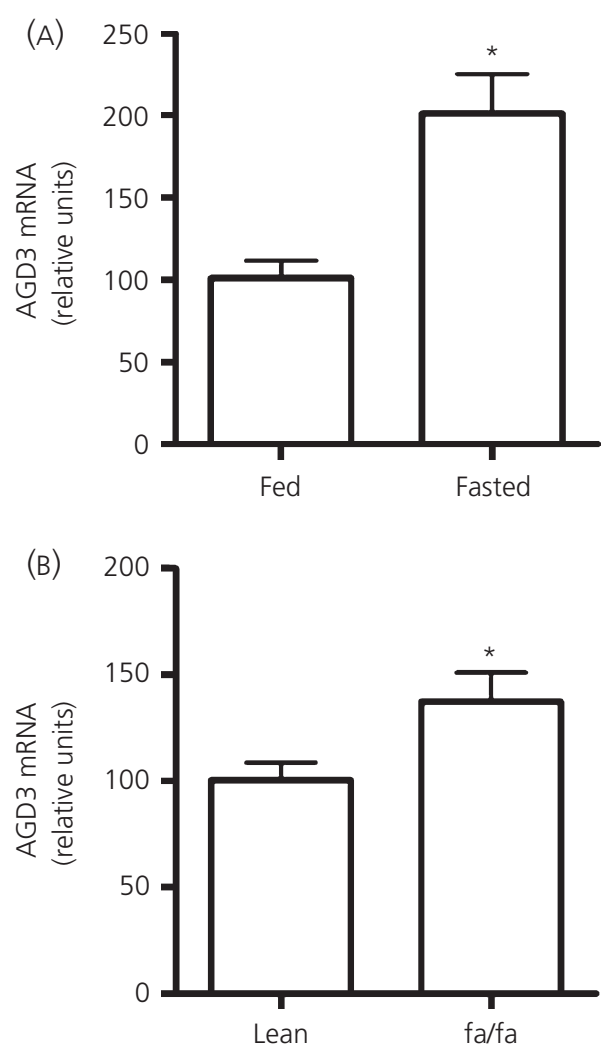

Fig. 1. Up-regulation of adipogenesis down-regulating transcript 3 (AGD3) mRNA in arcuate nucleus neurones by fasting and obesity. (A) AGD3 mRNA was significantly increased in fasted rats compared to fed rats $(n=5)$. (B) Compared with lean Zucker rats, AGD3 mRNA was significantly increased in obese Zucker rats (fa/fa) $(n=5)$. AGD3 mRNA levels were measured by a quantitative polymerase chain reaction. ${ }^{*} P<0.05$.

\section{Identifying the sequence of the hypothetical protein}

A rat hypothetical protein was produced, derived from this putative sequence, and an antibody directed against the hypothetical protein was created. The antibody recognised a single band when AGD3pcDNA3.1 was transfected into HEK293 cells, and recognised endogenous AGD3 protein in CACO-2 cells, SK-N-AS cells and the rat hypothalamus (Fig. 2A). By using this antibody, we examined the protein expression of $A G D 3$ in rat $A R C$, and the results showed that AGD3 protein significantly increased in fasted rats (Fig. 2B).

Three anti-AGD3 siRNAs were designed and transfected into CACO-2 cells. As shown in Fig. 3(A), AGD3 mRNA expression was inhibited by $90 \%$. AGD3 protein expression was decreased to a similar degree (Fig. 3B).

The antibody produced against the hypothetical protein was used to pull down AGD3 protein from CACO-1 cells. The bands that were recognised by the antibody on western blotting were used to identify proteins (Fig. 4A). Protein identification by mass spectrometry revealed the presence of the putative protein, over-expressed in colon carcinoma (OCC-1_HUMAN; Uniprot accession number 08TAD7) in samples 1 and 2 with 100\% probability at the protein level. The unambiguous identification of AGD3 is based on the
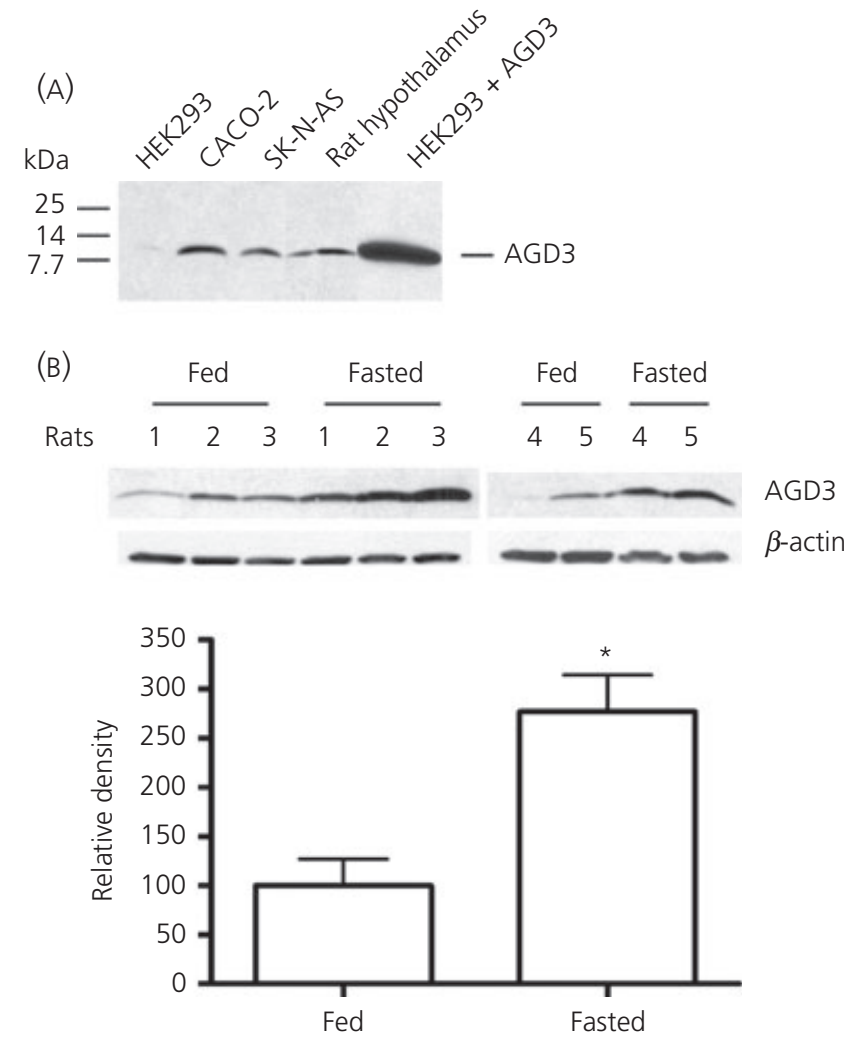

Fig. 2. (A) Specificity of adipogenesis down-regulating transcript 3 (AGD3) antibody. Various cell lines and rat hypothalamus were lysed with lysis buffer and supernatants were detected by western blotting using the anti-AGD3 antibody. This antibody detected a strong band in human embryonic kidney (HEK) cells transfected with AGD3-pcDNA3.1, whereas no detectable signal was found in HEK cells without transfection. AGD3 is also present in colorectal adenocarcinoma (CACO-2) cells, neuroblastoma (SK-N-AS) cells and the rat hypothalamus. (B) Up-regulation of AGD3 protein expression in the arcuate nucleus by fasting. AGD3 was measured by western blotting using the anti-AGD3 antibody. Each group comprised five animals. ${ }^{*} \mathrm{P}<0.05$.

observation of seven unique, well sequenced peptides (eight unique spectra and 11 total spectra) assigned to AGD3 in sample 1. In sample 2, three unique, well sequenced peptides (four unique spectra and seven total spectra) were assigned to AGD3. The sequence coverage for AGD3 is $86 \%$ in sample 1, and is $56 \%$ in sample 2. The identified sequence is shown in Figure 4(B).

\section{Distribution of AGD3 in rat brain}

\section{In situ hybridisation}

Figure 5 shows a photomicrograph of the neuroanatomical distribution of AGD3 mRNA. AGD3 mRNA was detected in several areas of the hypothalamus, including the $A R C$, and ventromedial nucleus (VMH). AGD3 mRNA was also observed in the amygdala, including the central and basolateral subnuclei of the amygdala, as well as in the hippocampus and the somatic cortex.

Figure 6 shows a photomicrograph of double in situ hybridisation in the ARC. Eight brain sections that cover the anterior and 


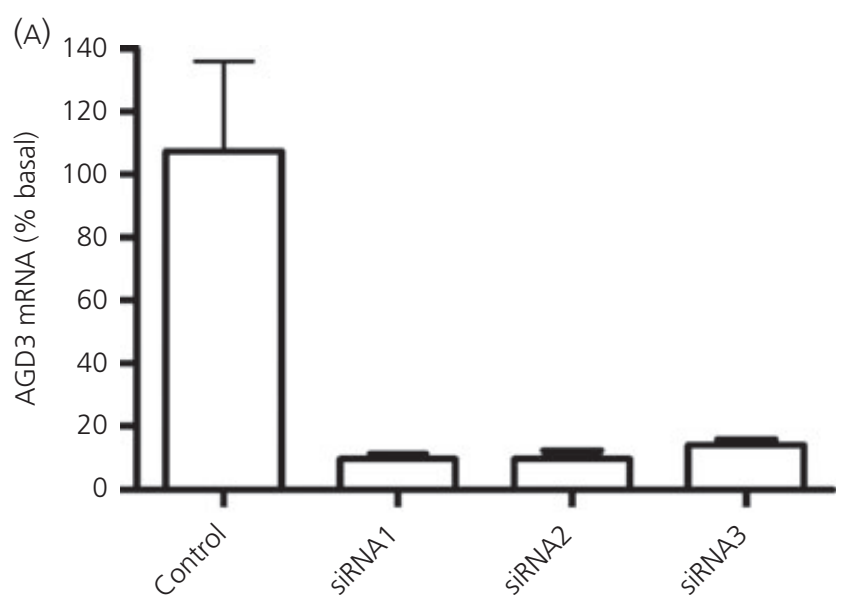

(B)

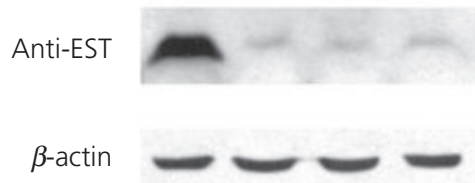

Fig. 3. Three small interfering (si)RNAs against different domains of adipogenesis down-regulating transcript 3 (AGD3) were transfected into colorectal adenocarcinoma (CACO-2) cells. These siRNAs inhibited AGD3 mRNA (A), resulting in decreased AGD3 protein expression (B). EST, expressed sequence tag.

(A)

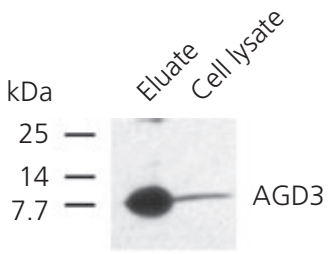

(B)

\section{G C GNSTATS A GA GQ G PA GA \\ AKDVTEESVT EDDKRRNYGG \\ VYVGLPSEAV NMVSSQTKTVRKN}

Fig. 4. Identification of human adipogenesis down-regulating transcript 3 (AGD3) protein sequence. (A) Anti-AGD3 antibody was linked to agarose beads to pull down AGD3 protein. Eluates were separated by electrophoresis and the bands, which were confirmed by western blotting, were analysed by mass spectrometry. (B) Mass spectrometry identified the presence of AGD3 protein. The sequence depicted is a human hypothetical protein sequence, with the highlighted portions confirmed by mass spectrometry.

middle portions of the hypothalamus from Bregma -2.12 to $-3.8 \mathrm{~mm}$ were hybridised and counted for each co-localisation. Both POMC and NPY neurones have clusters of silver grains over them. Each slice has similar co-localisation. Overall, $85 \pm 12 \%$ of POMC and $44 \pm 9 \%$ of NPY neurones express AGD3.

\section{Immunohistochemistry}

Immunohistochemistry revealed that AGD3 protein is present in the $A R C, V M H$, amygdala, hippocampus and somatic cortex, correspond-

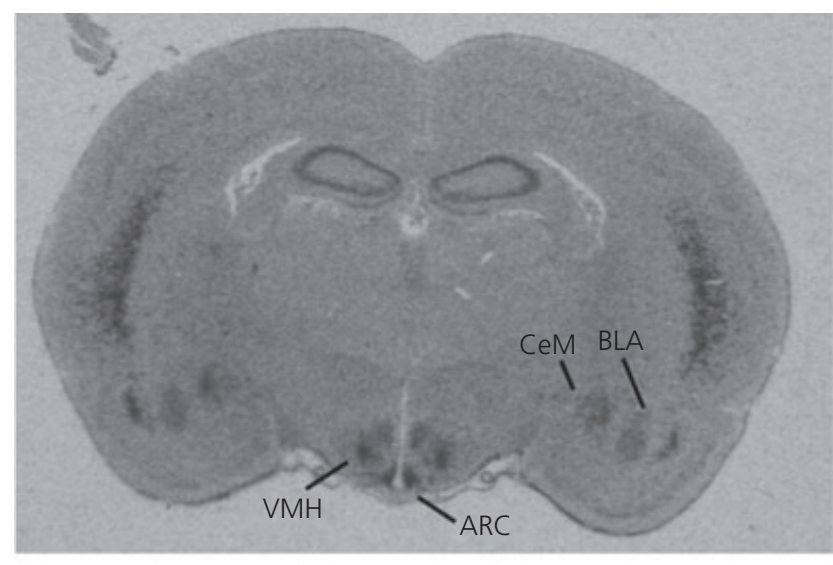

Antisense probe

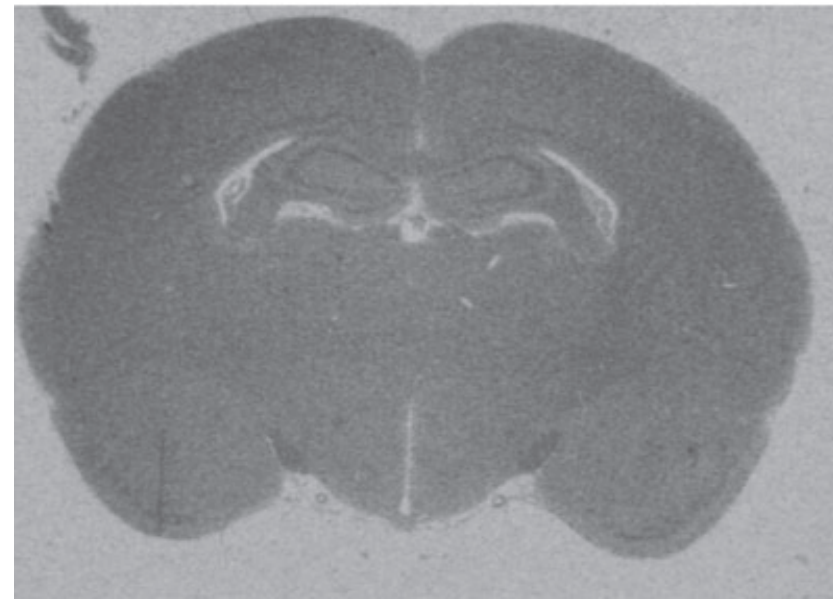

Sense probe

Fig. 5. In situ hybridisation of adipogenesis down-regulating transcript 3 (AGD3) in rat brain. AGD3 mRNA is distributed in the arcuate nucleus (ARC), ventromedial hypothalamus (VMH), amygdaloid nuclei (CeM and BLA), hippocampus and somatic cortex.

ing with its mRNA distribution. Double immunohistochemistry showed that AGD3 is present in POMC neurones from both the ARC and amygdala (Fig. 7).

\section{Interaction with IRS4}

To identify molecules that interact with AGD3, myc-AGD3 pcDNA3.1 was transfected into HEK293 cells. Cell lysates were incubated with anti-Myc agarose beads or protein $A$ beads plus normal immunoglobulin G. The gel bands in AGD3 lanes that were missing bands in control were sent to identify constituents by mass spectrometry analysis. IRS4 was pulled down by anti-AGD3 antibody. To confirm this interaction, we performed co-immunoprecipitation experiments using HEK293 cells that have endogenous IRS4 expression. Myc-AGD3 was expressed in HEK293 cells. The cell lysates were incubated with anti-Myc antibody conjugated to agarose. Anti-IRS4 antibody was used to detect endogenous IRS4. As shown in Fig. 8(A), endogenous IRS4 was co-precipitated 
POMC (purple) and AGD3 (silver grains)

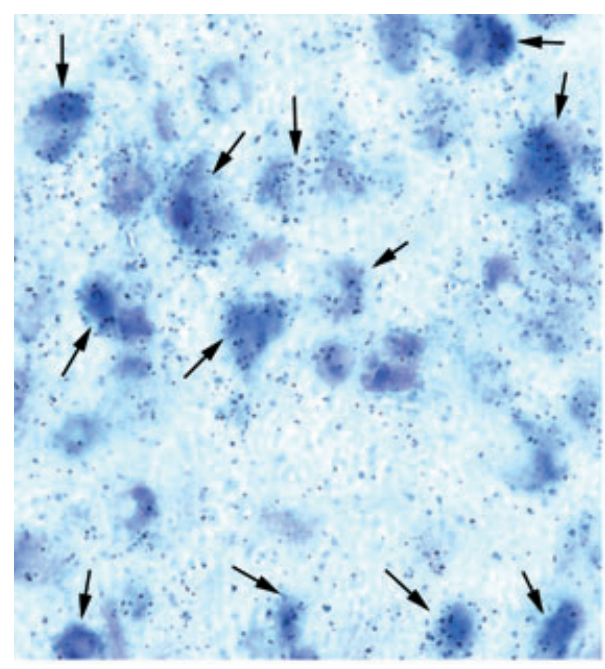

NPY (purple) and AGD3 (silver grains)

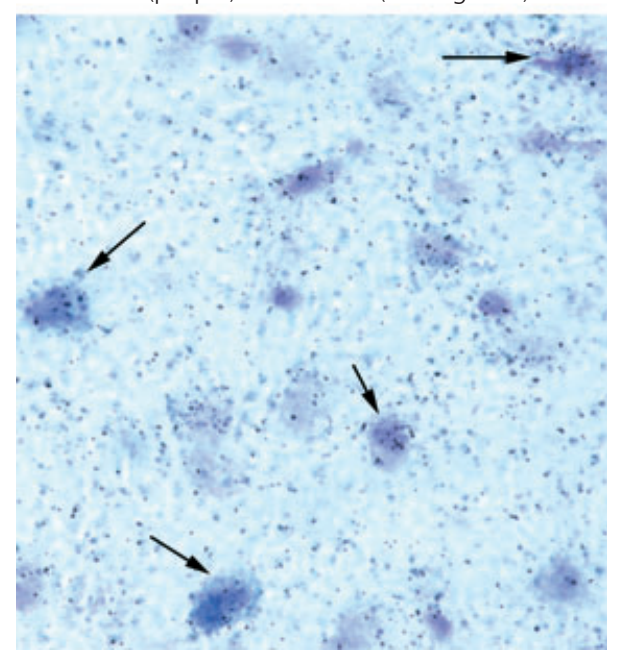

Fig. 6. Double in situ hybridisation of adipogenesis down-regulating transcript 3 (AGD3) in the rat arcuate nucleus. AGD3 mRNA is co-localised with pro-opiomelanocortin (POMC) (upper panel) and neuropeptide Y (NPY) neurones (lower panel). Blue represents digoxigenin-labelled POMC and NPY neurones. Silver grains indicate AGD3 mRNA.

with AGD3; IRS4 was pulled down by anti-AGD3 antibody from rat hypothalamus tissue (Fig. 8B). To examine whether IRS4 can pull down AGD3, HEK293 cells were transfected Flag-IRS4-CMV plasmid along with myc-AGD3 pcDNA3.1 DNA. AGD3 was pulled down by IRS4 (Fig. 8c).

\section{Involvement of the ADG3 in insulin signalling}

Because AGD3 interacts with IRS4, which is an element in the insulin signalling pathway, we investigated the role of AGD3 in Akt phosphorylation, a component of insulin signalling. SK-N-AS cells were transfected with AGD3 and Akt phosphorylation was examined. AGD3 expression in SK-N-AS cells significantly enhanced insulin-stimulated Akt phosphorylation (Thr308) (Fig. 9A). When the SK-N-AS cells were transfected with AGD3 siRNA for $72 \mathrm{~h}$, insulin-
ARC

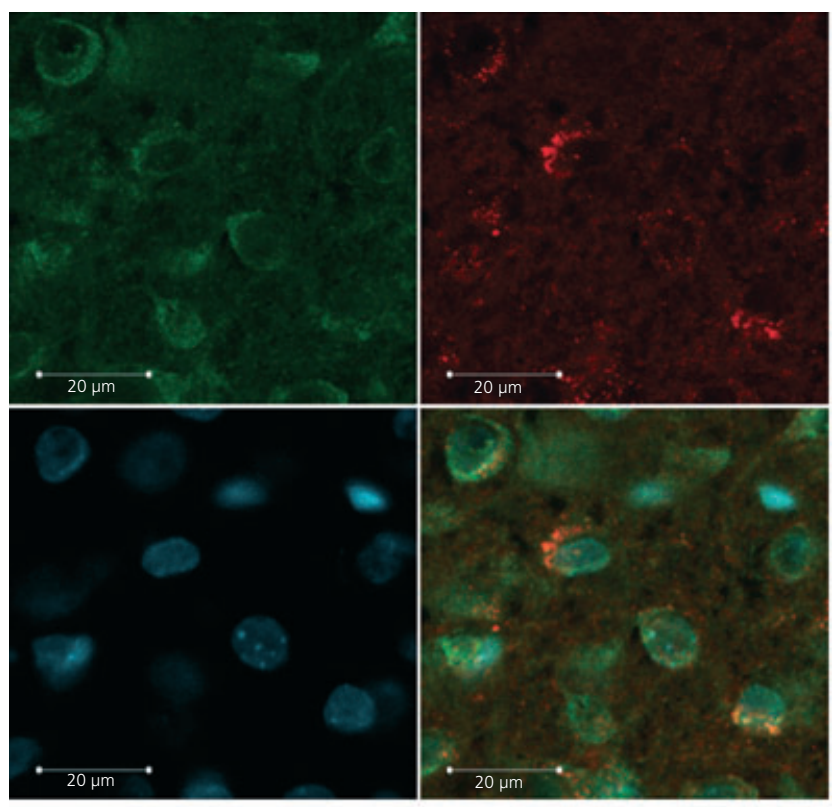

Amygdala

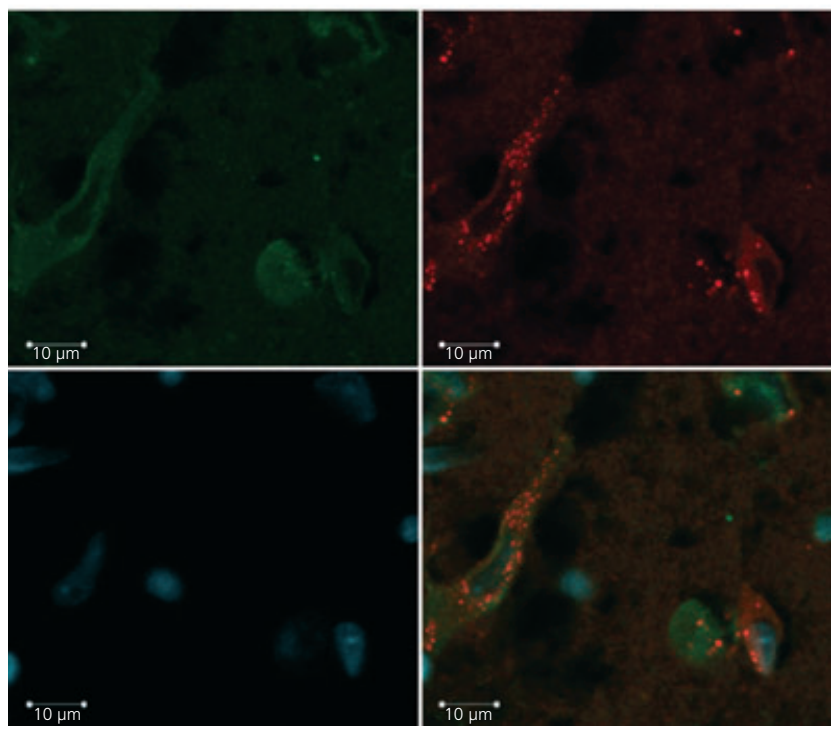

Fig. 7. Co-localisation of adipogenesis down-regulating transcript 3 (AGD3) with pro-opiomelanocortin (POMC) neurones in the arcuate nucleus (ARC) and amygdala. Confocal photomicrographs of dual staining of AGD3 (green) and POMC (red) shows the co-localisation of AGD3 with POMC. Nuclei are stained with 4',6-diamidino-2-phenylindole dihydrochloride.

stimulated Akt phosphorylation (Thr308) was significantly inhibited. The extent of inhibition was $28.6 \pm 6 \%$ (Fig. 9B).

Involvement in AMPK and mammalian target of rapamycin (mTOR) signalling pathway

The AMPK kinase and mTOR signalling systems are responsive to cellular energy status and are involved in hypothalamic adaptation to fasting and feeding. An AGD3 siRNA approach was used to 
(A) KEK293 cells

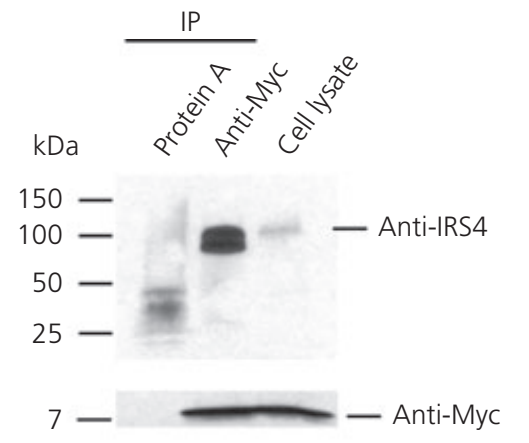

(B) Hypothalamus

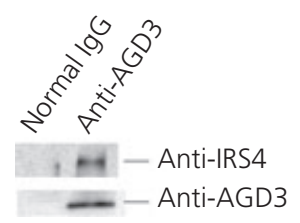

(c) HEK293 cells

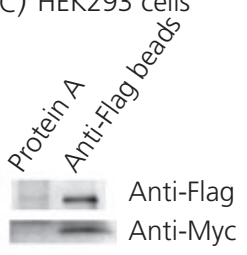

Fig. 8. Co-immunoprecipitation of adipogenesis down-regulating transcript 3 (AGD3) with insulin receptor substrate 4 (IRS4). (A) Myc-AGD3 vector was transfected into HEK293 cells, which have endogenous IRS4 expression. Cell lysates were immunoprecipitated with anti-Myc antibody-conjugated agarose and blotted with anti-IRS4 antibody. (B) Normal rabbit immunoglobulin (Ig)G or anti-AGD3 antibody were incubated with protein A beads and rat hypothalamus tissue lysate. IRS4 was detected by anti-IRS4. (c) Flag-IRS4 and Myc-AGD3 vectors were co-transfected into HEK293 cells. Cell lysates were precipitated with anti-Flag M2 beads and blotted with anti-Myc antibody. The membranes then were stripped and re-blotted with anti-Flag antibody. Protein A beads were used as a negative control.

determine whether AGD3 interacted with these pathways. As shown in Fig. 10, transfection of GT1-1 cells with AGD3 siRNA affected these pathways differentially, decreasing AMPK phosphorylation and increasing the expression of pP70 and pS6, which are the downstream signal molecules in the mTOR signal pathway (14). This result indicates that $A G D 3$ up-regulates phosphorylation of AMPK and inhibits mTOR pathway.

\section{Discussion}

The present study describes a novel 63-amino acid peptide, AGD3, which is expressed in areas of the brain related to the control of ingestive behaviour that interact with energy signalling pathways. This conclusion is supported by distinct observations: (i) AGD3 mRNA is regulated by fasting and feeding in the hypothalamic $A R C$; (ii) AGD3 encodes a novel 63-amino acid protein; (iii) the protein interacts specifically with insulin receptor substrate 4 ; (iv) the protein is expressed in regions of the rat brain relevant to feeding behaviour; ( $v$ ) there is a high degree of overlap with brain areas that also express insulin receptor substrate 4 ; and (vi) the protein affects Akt, AMPK and mTOR-dependent signalling in vitro.

The novel protein characterised in the present study is derived from a gene of approximately $1.2 \mathrm{kbp}$ that has a high degree of homology with the human OCC-1 gene first described by Pibouin
(A)
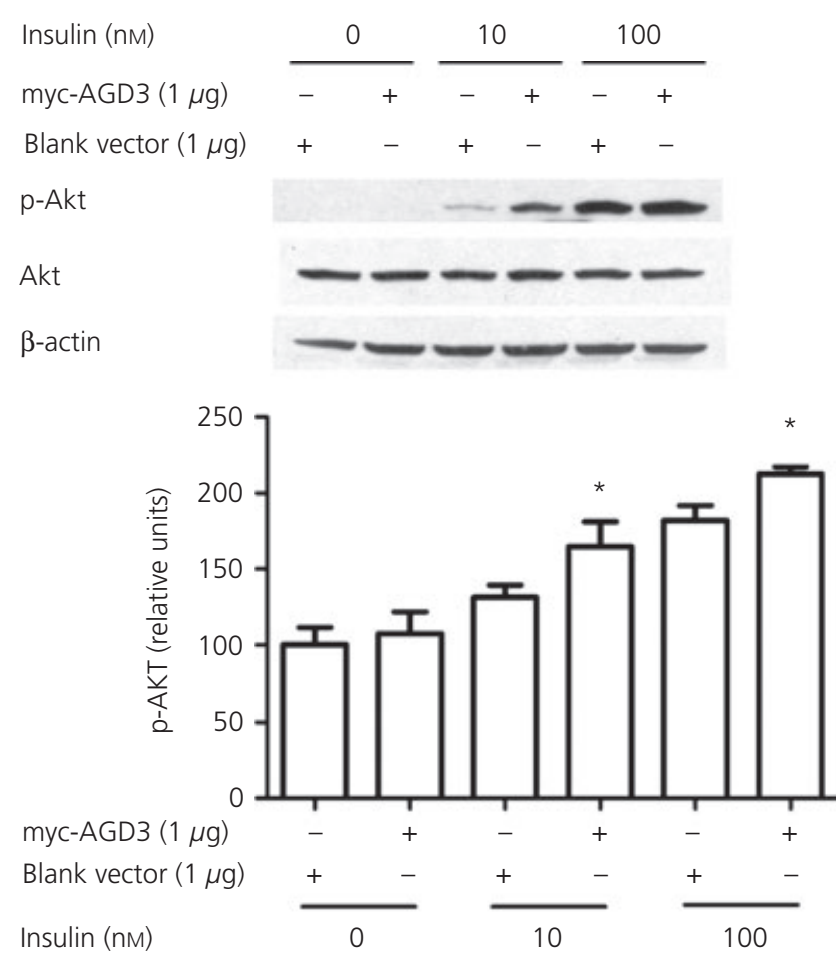

(B)

\begin{tabular}{|c|c|c|c|c|}
\hline Insulin (nм) & 0 & & & 00 \\
\hline Control RNA (17 nм) & + & - & + & - \\
\hline siRNA2 (17 nм) & - & + & - & + \\
\hline $\mathrm{p}-\mathrm{Akt}$ & 13 & wes & $=$ & 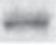 \\
\hline & $=$ & 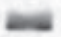 & $=$ & 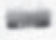 \\
\hline
\end{tabular}

Fig. 9. Enhancement of insulin-stimulated Akt phosphorylation by adipogenesis down-regulating transcript 3 (AGD3). (A) Neuroblastoma (SK-N-AS) cells were transfected with myc-AGD3 pcDNA3.1. After $48 \mathrm{~h}$ of transfection, 10\% serum Dulbecco's modified Eagle's medium (DMEM) was replaced with serum-free DMEM containing $0.1 \%$ bovine serum albumin (BSA) and incubated for another $2 \mathrm{~h}$. Then, cells received insulin treatment for $20 \mathrm{~min}$. (B) SK-N-AS cells were transfected with AGD3 small interfering RNA2 for $72 \mathrm{~h}$. Cells were incubated with $0.1 \%$ BSA DMEM for $4 h$, then, treated with insulin for 20 min. Phosphorylation of Akt was detected by phospho-Akt antibody (Thr308).

et al. (11). These investigators identified a novel human cDNA overexpressed in a colon carcinoma cell line derived from a 29-year-old woman with hereditary nonpolyposis colorectal cancer. The gene is composed of six exons and is located in the q24.1 region of human chromosome 12. Elevated levels of OCC-1 mRNA were observed in three of eight colon carcinomas relative to normal mucosa from the affected patient. However, no specific OCC-1 proteins were detected.

The peptide characterised in the present study has also been referred to as adipogenesis down-regulating transcript 3 (ADG3) by Kikuchi et al. (12). These investigators used a panel of human bone marrow-derived mesenchymal stem cells (hMSC) and measured 


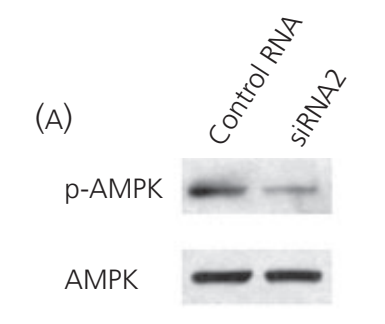

(B)
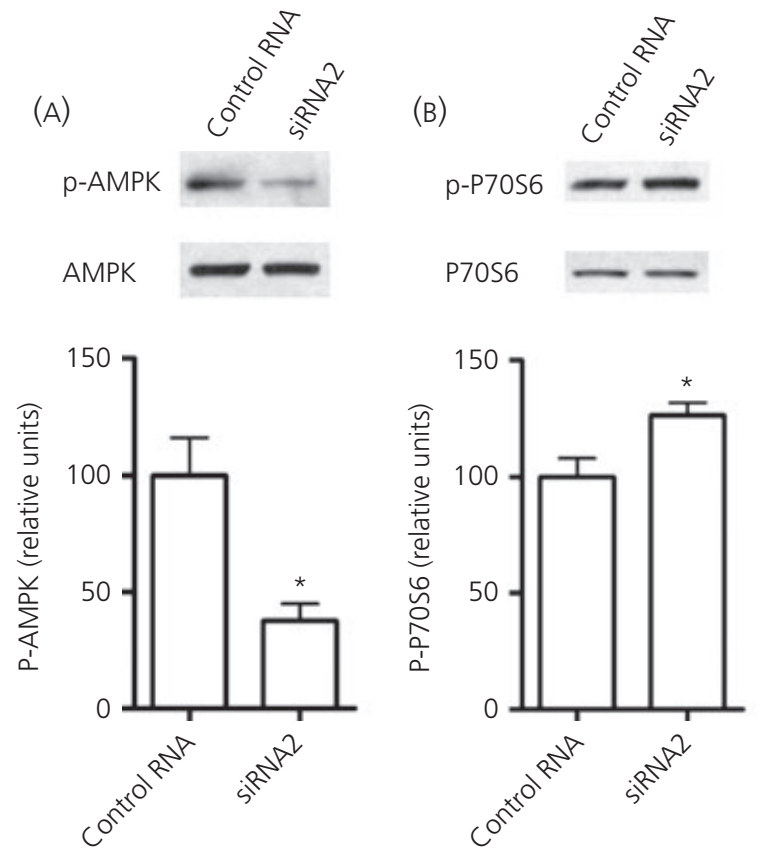

(c)
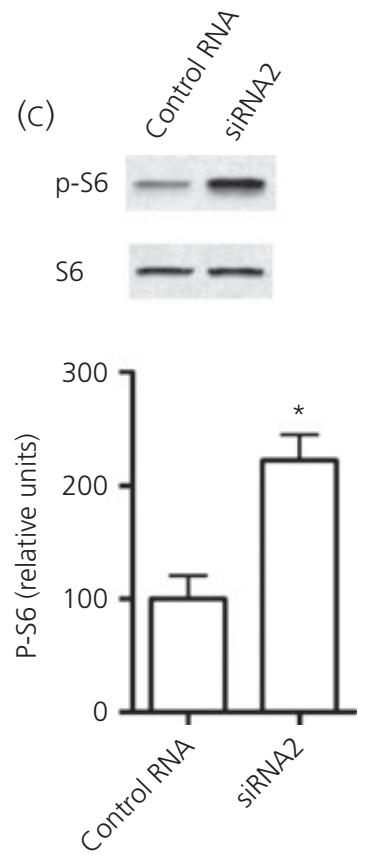

Fig. 10. The role of adipogenesis down-regulating transcript 3 (AGD3) in AMP-activated protein kinase (AMPK) and mammalian target of rapamycin (mTOR) signalling. Small interfering (si)RNA against AGD3 mRNA was transfected in mouse hypothalamus (GT1-1) cells for $72 \mathrm{~h}$, and then starved for $4 \mathrm{~h}$ in serumfree Dulbecco's modified Eagle's medium medium containing $1 \mathrm{~mm}$ glucose. Cell lysates were lysed with lysis buffer, and supernatants were used to detect phosphorylation of AMPK (A), P70 S6 kinase (B) and S6 (C) by western blotting.NC1 negative control RNA was used as a control.

adipogenic and osteogenic induction in response to an array of transcripts of unknown function. AGD3 strongly inhibited adipogenic induction in response to a medium containing insulin, dexamethasone, indomethacin and isobutylmethylxanthine. Polyclonal antibody was prepared against the hypothetical human AGD3 protein. Western blotting confirmed that ADG3 expression was reduced when hMSC were treated with AGD3 siRNAs, implying that an intracellular peptide was produced by this transcript.

The present study is the first to describe the amino acid sequence and localisation of this transcript in normal adult rat brain tissues. AGD3 mRNA was detected in several regions of the brain that are relevant to feeding behaviour. Within the hypothalamus, these include the ARC, and VMH. The ARC is a well-recognised centre for the integration of peripheral and central signals that regulate appetite and metabolism (5). ARC neurones express receptors for a variety of circulating factors, including insulin $(15,16)$. AGD3 mRNA was also observed in the amygdala, the hippocampus and the somatic cortex. The amygdala is primarily involved in emotions such as fear, anger and pleasure, as well as memory $(17,18)$. The amygdala may also be involved in motivation and reward associated with eating $(5,19)$. The amygdala sends projections to the lateral hypothalamic area, and forms part of a circuit linked to initiation of feeding (20-22).

In both the hypothalamus and amygdala, AGD3 mRNA was detected in neurones that were positive for POMC peptide. The best described anorexigenic neurones within the ARC express POMC and CART. POMC neurones in the ARC play a key role in the regulation of energy homeostasis. Two cleavage products of the POMC precur- sor, $\alpha$ and $\beta$-melanocyte-stimulating hormones, inhibit food intake and increase energy expenditure via melanocortin receptor subtypes 3 and 4 in the ARC, PVN and lateral hypothalamus $(4,5)$. Efferent projections from POMC/CART neurones to secondary energy homeostatic neurones in intra- and extra-hypothalamic sites are key features of energy homeostatic circuitry.

The AGD3 distribution in brain tissue showed a substantial overlap with that of IRS4. We have previously reported that IRS4 mRNA is expressed in the ARC, VMH, dorsomedial nucleus, lateral hypothalamus and amygdala (13). IRS proteins represent a family of adapter proteins that play central roles in signal transduction by insulin, insulin-like growth factor 2 and cytokines such as leptin (23-25). Although the different IRS proteins vary in size, they share structural and functional characteristics. The $\mathrm{NH}_{2}$-terminal of each protein contains a homologous pleckstrin homology domain and a phosphotyrosine binding region that mediate interactions with insulin receptors. The $\mathrm{COOH}$-terminal portion of each protein contains tyrosine phosphorylation sites $(26,27)$. Tyr-phosphorylated IRS proteins serve as signalling scaffolds that propagate hormone action through the binding of Src homology 2 domain-containing proteins.

Although IRS4 is considered to have a role in signal transduction similar to other members of the IRS family, the tissue distribution of IRS4 is substantially different from that of other insulin receptor substrates. The expression of IRS4 mRNA is restricted to the hypothalamus in the brain, suggesting that IRS4 might be involved in the regulation of energy homeostasis (28). In a previous study, we demonstrated that IRS4 is expressed in POMC neurones of the ARC. 
The expression of IRS4 in POMC neurones implies that IRS4 may function to mediate the action of insulin in these neurones.

Because IRS4 is a component of insulin signalling, the effect of AGD3 expression on insulin signalling was examined. We investigated the role of AGD3 in Akt phosphorylation using SK-N-AS cells that were transfected with AGD3. AGD3 expression in SK-N-AS cells significantly enhanced insulin-stimulated Akt phosphorylation (Thr308).

The AMPK kinase and mTOR signalling systems are responsive to changes in organism and cellular energy status and have been studied extensively in the hypothalamic control of feeding. An AGD siRNA approach was used to determine whether AGD3 interacted with these pathways. In GT1-1 cells treated with AGD3 siRNA, AMPK phosphorylation was suppressed, whereas phosphorylation of P70 and S6, comprising molecules in the mTOR signal pathway, was increased.

In summary, AGD3 functions within the hypothalamic ARC as a component of the insulin signalling pathway. The localisation of AGD3 within both POMC-positive neurones and NPY-expressing neurones, which have generally opposing actions in the regulation of ingestive behaviour, further suggests that AGD3 may have a central role in sensing organismal energy states. The regulation of the AMPK and mTOR pathways by AGD3 is consistent with this possibility and represents an area for further investigation.

\section{Acknowledgements}

The work was supported by NIH grants 2R01DK054032 and 5R37DK043225-20.

Received 16 March 2012,

revised 16 July 2012,

accepted 24 August 2012

\section{References}

1 Lam TK, Schwartz GJ, Rossetti L. Hypothalamic sensing of fatty acids. Nat Neurosci 2005; 8: 579-584.

2 Sandoval D, Cota $D$, Seeley RJ. The integrative role of CNS fuel-sensing mechanisms in energy balance and glucose regulation. Annu Rev Physiol 2008; 70: 513-535.

3 Woods SC, Lutz TA, Geary N, Langhans W. Pancreatic signals controlling food intake; insulin, glucagon and amylin. Philos Trans $R$ Soc Lond $B$ Biol Sci 2006; 361: 1219-1235.

4 Schwartz MW, Woods SC, Porte D Jr, Seeley RJ, Baskin DG. Central nervous system control of food intake. Nature 2000; 404: 661-671.

5 Morton GJ, Cummings DE, Baskin DG, Barsh GS, Schwartz MW. Central nervous system control of food intake and body weight. Nature 2006; 443: 289-295.

6 Li JY, Kuick R, Thompson RC, Misek DE, Lai YM, Liu YQ, Chai BX, Hanash SM, Gantz I. Arcuate nucleus transcriptome profiling identifies ankyrin repeat and suppressor of cytokine signalling box-containing protein 4 as a gene regulated by fasting in central nervous system feeding circuits. J Neuroendocrinol 2005; 17: 394-404.

7 Li JY, Lescure PA, Misek DE, Lai YM, Chai BX, Kuick R, Thompson RC, Demo RM, Kurnit DM, Michailidis G, Hanash SM, Gantz I. Food deprivation-induced expression of minoxidil sulfotransferase in the hypothalamus uncovered by microarray analysis. J Biol Chem 2002; 277: 90699076.
8 Carninci $P$, Kasukawa $T$, Katayama $S$, Gough J, Frith MC, Maeda $N_{1}$ Oyama R, Ravasi T, Lenhard B, Wells C, Kodzius R, Shimokawa K, Bajic VB, Brenner SE, Batalov S, Forrest ARR, Zavolan M, Davis MJ, Wilming

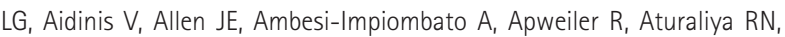
Bailey TL, Bansal M, Baxter L, Beisel KW, Bersano T, Bono H, Chalk AM, Chiu KP, Choudhary V, Christoffels A, Clutterbuck DR, Crowe ML, Dalla E, Dalrymple BP, de Bono B, Della Gatta G, di Bernardo D, Down T, Engstrom $P$, Fagiolini $M$, Faulkner $G$, Fletcher $C F$, Fukushima $T$, Furuno $M$, Futaki S, Gariboldi M, Georgii-Hemming P, Gingeras TR, Gojobori T, Green RE, Gustincich S, Harbers M, Hayashi Y, Hensch TK, Hirokawa N, Hill $D$, Huminiecki $L$, lacono $M$, Ikeo $K$, Iwama $A$, Ishikawa $T$, Jakt $M$, Kanapin A, Katoh M, Kawasawa Y, Kelso J, Kitamura H, Kitano H, Kollias G, Krishnan SPT, Kruger A,Kummerfeld SK, Kurochkin IV, Lareau LF, Lazarevic D, Lipovich L, Liu J, Liuni S, McWilliam S, Madan Babu M, Madera $M$, Marchionni L, Matsuda $H_{1}$ Matsuzawa $S$, Miki $H_{1}$ Mignone $F_{\text {, }}$ Miyake S, Morris K, Mottagui-Tabar S, Mulder N, Nakano N, Nakauchi H, $\mathrm{Ng}$ P, Nilsson R, Nishiguchi $S$, Nishikawa $S$, Nori F, Ohara O, Okazaki $Y$, Orlando V, Pang KC, Pavan WJ, Pavesi G, Pesole G, Petrovsky N, Piazza S, Reed J, Reid JF, Ring BZ, Ringwald M, Rost B, Ruan Y, Salzberg SL, Sandelin A, Schneider C, Schonbach C, Sekiguchi K, Semple CAM, Seno $S$, Sessa L, Sheng $Y$, Shibata $Y$, Shimada $H$, Shimada K, Silva D, Sinclair B, Sperling S, Stupka E, Sugiura K, Sultana R, Takenaka Y, Taki K, Tammoja K, Tan SL, Tang S, Taylor MS, Tegner J, Teichmann SA, Ueda HR, van Nimwegen E, Verardo R, Wei CL, Yagi K, Yamanishi H, Zabarovsky E, Zhu S, Zimmer A, Hide W, Bult C, Grimmond SM, Teasdale RD, Liu ET, Brusic V, Quackenbush J, Wahlestedt C, Mattick JS, Hume DA, Kai C, Sasaki D, Tomaru Y, Fukuda S, Kanamori-Katayama M, Suzuki M, Aoki J, Arakawa T, lida J, Imamura K, Itoh M, Kato T, Kawaji H, Kawagashira N, Kawashima T, Kojima M, Kondo $S$, Konno $H_{1}$ Nakano $K$, Ninomiya $N$, Nishio T, Okada M, Plessy C, Shibata K, Shiraki T, Suzuki S, Tagami M, Waki K, Watahiki A, Okamura-Oho Y, Suzuki H, Kawai J, Hayashizaki Y, Consortium F, Group RGER, Genome Science G. The transcriptional landscape of the mammalian genome. Science 2005; 309: 1559-1563. Erratum appears in Science. 2006 Mar 24;311(5768):1713.

9 Mattick JS, Makunin IV. Non-coding RNA. Hum Mol Genet 2006; 15: R17-R29.

10 Knowling S, Morris KV. Non-coding RNA and antisense RNA. Nature's trash or treasure? Biochimie 2011; 93:1922-1927

11 Pibouin L, Villaudy J, Ferbus D, Muleris M, Prosperi MT, Remvikos Y, Goubin G. Cloning of the mRNA of overexpression in colon carcinoma1: a sequence overexpressed in a subset of colon carcinomas. Cancer Genet Cytogenet 2002; 133: 55-60.

12 Kikuchi K, Fukuda M, Ito T, Inoue M, Yokoi T, Chiku S, Mitsuyama T, Asai $K_{1}$ Hirose T, Aizawa Y. Transcripts of unknown function in multiplesignaling pathways involved in human stem cell differentiation. Nucleic Acids Res 2009; 37: 4987-5000.

13 Li J-Y, Chai B, Zhang W, Wu X, Zhang C, Fritze D, Xia Z, Patterson C, Mulholland MW. Ankyrin repeat and SOCS box containing protein 4 (Asb-4) colocalizes with insulin receptor substrate 4 (IRS4) in the hypothalamic neurons and mediates IRS4 degradation. BMC Neurosci 2011; 12: 95.

14 Dennis PB, Jaeschke A, Saitoh M, Fowler B, Kozma SC, Thomas G. Mammalian TOR: a homeostatic ATP sensor. Science 2001; 294: 1102-1105.

15 Niswender KD, Baskin DG, Schwartz MW. Insulin and its evolving partnership with leptin in the hypothalamic control of energy homeostasis. Trends Endocrinol Metab 2004; 15: 362-369.

16 Maejima $Y$, Kohno D, Iwasaki $Y$, Yada T. Insulin suppresses ghrelininduced calcium signaling in neuropeptide $Y$ neurons of the hypothalamic arcuate nucleus. Aging (Albany NY) 2011; 3: 1092-1097.

17 LeDoux J. The amygdala. Curr Biol 2007; 17: R868-R874.

18 Cardinal RN, Parkinson JA, Hall J, Everitt BJ. Emotion and motivation: the role of the amygdala, ventral striatum, and prefrontal cortex. Neurosci Biobehav Rev 2002; 26: 321-352. 
19 Baxter MG, Murray EA. The amygdala and reward. Nat Rev Neurosci 2002; 3: 563-573.

20 Petrovich GD, Setlow B, Holland PC, Gallagher M. Amygdalo-hypothalamic circuit allows learned cues to override satiety and promote eating. J Neurosci 2002; 22: 8748-8753.

21 Petrovich GD, Canteras NS, Swanson LW. Combinatorial amygdalar inputs to hippocampal domains and hypothalamic behavior systems. Brain Res Brain Res Rev 2001; 38: 247-289.

22 Elmquist JK, Elias CF, Saper CB. From lesions to leptin: hypothalamic control of food intake and body weight. Neuron 1999; 22: 221-232.

23 White MF. IRS proteins and the common path to diabetes. Am J Physiol Endocrinol Metab 2002; 283: E413-E422.

24 Wauman J, De Smet AS, Catteeuw D, Belsham D, Tavernier J. Insulin receptor substrate 4 couples the leptin receptor to multiple signaling pathways. Mol Endocrinol 2008; 22: 965-977.
25 Duan C, Li M, Rui L. SH2-B promotes insulin receptor substrate 1 (IRS1)- and IRS2-mediated activation of the phosphatidylinositol 3-kinase pathway in response to leptin. J Biol Chem 2004; 279: 4368443691.

26 Yenush L, Zanella C, Uchida T, Bernal D, White MF. The pleckstrin homology and phosphotyrosine binding domains of insulin receptor substrate 1 mediate inhibition of apoptosis by insulin. Mol Cell Biol 1998; 18: 6784-6794.

27 Yenush L, White MF. The IRS-signalling system during insulin and cytokine action. BioEssays 1997; 19: 491-500.

28 Numan S, Russell DS. Discrete expression of insulin receptor substrate-4 mRNA in adult rat brain. Brain Res Mol Brain Res 1999; 72: 97-102. 